\title{
Enunciación
}

http://revistas.udistrital.edu.co/ojs/index.php/enunc

\section{Expectativas sobre la escritura en la educación superior: el caso de un proyecto colombiano interinstitucional}

\author{
Expectations on Higher Education Writing: The Case of a Colombian \\ Inter-Institutional Project
}

\author{
Elizabeth Narváez Cardona ${ }^{1}$
}

Para citar este artículo: Narváez E., (2014). Expectativas sobre la escritura en la educación superior: el caso de un proyecto colombiano interinstitucional. Enunciación, 19(1), 10-25.

Recibido: 25-abril-2014 / Aprobado: 12-julio-2014

\section{Resumen}

A fin de aportar a la sistematización de los esfuerzos generados en Colombia y de añadir narrativas sobre los mismos a las tradiciones internacionales desarrolladas en el campo de los estudios de la escritura, este artículo reporta un mapa de expectativas sobre la escritura en la educación superior emergentes de reportes escritos por investigadores para un proyecto colombiano interinstitucional desarrollado entre 2009 y 2011 en el cual participé. El análisis de seis reportes institucionales de tres universidades distintas ofrece una descripción de problemas y soluciones que podrían representar agendas de práctica y de investigación que se están adelantando en el país y se pueden comparar con otras agendas internacionales. Los movimientos sobre la escritura a través del currículo (WAC) y la escritura en las disciplinas (WID), originalmente desarrollados en EEUU, serían los paradigmas desde los cuales se enmarca la mayoría de las expectativas inferidas del análisis de los reportes.

\begin{abstract}
With the goal of systematizing efforts generated in Colombia and adding narratives into international data on Writing Studies, this paper brings a map of expectations on higher education writing that emerge from research reports of a Colombian inter-institutional project conducted between 2009 and 2011, in which I took part. The analysis of six institutional reports from three different institutions provides a description of problems and solutions that might represent agendas of practice and research developed in the country to be compared with other international agendas. The advancements on Writing Across the Curriculum (WAC) and Writing In Disciplines (WID), originally developed in the US, seem to be the paradigms in which most the expectations inferred from analysis are framed.
\end{abstract}

Keywords: Writing across the Curriculum (WAC), Writing in Disciplines (WID), research, institutional reports

Palabras clave: escritura a través del currículo (WAC), escritura en las disciplinas (WID), investigación, reportes institucionales

1 Adelanta estudios de doctorado en Educación con énfasis en estudios de la escritura en la Universidad de California, Santa Bárbara, gracias a una beca cooperativa entre Fulbright y Colciencias; estudios en lingüística, didáctica del español como lengua materna y fonoaudiología; profesora del Departamento de Lenguaje de la Facultad de Comunicación Social de la Universidad Autónoma de Occidente (Cali, Colombia). Correo electrónico: enarvaez@uao.edu.co 


\section{INTRODUCCIÓN}

El campo de los estudios de la escritura en la educación superior se ha desarrollado en diferentes partes del mundo bajo las características locales de los sistemas universitarios y las transformaciones educativas asociadas a ellos. Como resultado, las agendas de investigación y de intervención pedagógica del campo de la escritura en la educación superior han sido permeadas por dichos rasgos locales de las universidades.

En el caso británico las reformas educativas dieron acceso a nuevas poblaciones de estudiantes a la educación superior, quienes incorporaron diversidad cultural y lingüística. La descripción de prácticas de escritura de dicha población estudiantil, denominada "estudiantes no tradicionales", ha sido un foco de interés en algunos estudios (Lillis, 2003).

En Estados Unidos la investigación y enseñanza de la escritura en la educación superior se inició en 1960 como una tendencia pedagógica liderada por los departamentos de inglés; se desarrollaron movimientos conocidos como "la escritura a través del currículo" (WAC, por sus siglas en inglés) y la "escritura en las disciplinas" (WID, por sus siglas en inglés) (Russell, Lea, Parker y Donahue, 2009). Entre otros aspectos, dichos movimientos se encuentran a favor de que los profesores de las disciplinas se interesen por enseñar la escritura a sus estudiantes a fin de mejorar su formación disciplinar. Dado que el sistema universitario en EEUU para la formación de pregrado incluye un fuerte componente de educación general y no especializada o profesionalizante, el debate pedagógico sobre la enseñanza de la escritura se ha centrado en cómo desarrollar programas de escritura a lo largo de la formación de pregrado, así como las prácticas pedagógicas que les permitan a los estudiantes y profesores anticipar y reconocer la existencia de la escritura disciplinar, ligada a las formas de decir y pensar en los campos de conocimiento, a pesar de que los estudiantes no declaran una afiliación profesional o disciplinar desde el principio de la formación (Russell, 2003; 2013).
En el caso de Francia, algunas tradiciones de investigación han estudiado las relaciones entre los géneros académicos y las prácticas escritas. Algunos estudios han enfatizado que el análisis textual es insuficiente para revelar las complejidades de la escritura cuando es un objeto de enseñanza. En su lugar, se sugiere la incorporación de análisis de contexto; por ejemplo, características de las clases, valoraciones asociadas a la enseñanza, políticas institucionales y tradiciones disciplinares (Donahue, 2009).

En consecuencia, la diversidad de desarrollos en los estudios sobre la escritura en la educación superior también ha permitido caracterizar la coexistencia de prácticas escritas tradicionales e innovadoras. Algunos estudios argumentan que las trayectorias de los estudiantes como escritores en la educación superior están influenciadas por experiencias pedagógicas previas y la función explícita o implícita que la escritura ha tenido en ellas. Como resultado, estos estudios concluyen que aprender sobre la escritura en la educación superior implica dominar prácticas de lectura y escritura estables y tradicionales como aquellas asociadas a los artículos científicos, pero también prácticas menos estables y emergentes como el uso de los diarios escritos, producciones multimodales o el diseño de géneros académicos utilizando tecnologías digitales (Lillis, 2003; Thaiss, Zawacki y Myers, 2006).

Para el caso latinoamericano un proyecto en curso, en el que participo, titulado "Iniciativas de lectura y escritura en la educación superior, ILEES América Latina", ha construido un mapa de tendencias sobre la enseñanza y la investigación a partir de la sistematización de información recogida, entre otras estrategias, a través de una encuesta electrónica aplicada a una muestra intencional de profesores de Argentina, Brasil, Chile, Colombia, México, Puerto Rico y Venezuela (ILEES). Los resultados preliminares muestran que la tendencia en la región es la incorporación de los cursos de escritura de primer año, aunque hay una diversidad de iniciativas emergentes orientadas a 
la formación en escritura para audiencias distintas (estudiantes, profesores y audiencias no académicas) y en momentos distintos de la formación (pregrado, postgrado, formación de profesores): cursos de escritura de primer año $(19 \%)$, cursos en el currículo de las disciplinas $(13,6 \%)$, cursos de escritura después del primer año $(12,6 \%)$, talleres de postgrado $(12,1 \%)$, talleres para profesores universitarios $(9,8 \%)$, programas de escritura $(7,7 \%)$, centros de escritura $(5,1 \%)$, y experiencias en otras lenguas $(4,4 \%)$.

La documentación de otras iniciativas en la región confirma que la tendencia más difundida ha sido la enseñanza de la escritura durante el primer año. Dichos cursos se han orientado a partir de dos perspectivas distintas: en primer lugar la perspectiva remedial, cuyo propósito es abordar las carencias que los estudiantes transfieren de su educación previa; y en segundo lugar, la perspectiva de los géneros que sitúa la escritura en una disciplina y bajo una orientación de la escritura como proceso (borradores, revisiones y ediciones antes de la presentación del texto final). Esta documentación también ha destacado que las iniciativas emergentes en Latinoamérica han sido principalmente el resultado de los esfuerzos individuales de los profesores que las lideran, como consecuencia de sus convicciones y apoyos institucionales (Carlino, 2012).

En Colombia el campo de los estudios sobre la escritura en la educación superior emerge aproximadamente en el año 2000 (Uribe-Álvarez y Camargo-Martínez, 2011; Ortiz-Casallas, 2011). Una tradición investigativa justifica la importancia de este campo en la necesidad de encontrar soluciones ante las carencias de procesamientos cognitivos y lingüísticos de los estudiantes para escribir. Otra tendencia se centra en el estudio de prácticas de enseñanza en las disciplinas y en condiciones institucionales específicas (Quintero y Duque, 2011; Rincón y Gil, 2010; Rincón y Pérez, 2011).

A fin de aportar a la sistematización de los esfuerzos desarrollados en Colombia y de añadir narrativas sobre los mismos a las tradiciones internacionales desarrolladas, este artículo reporta un mapa de expectativas sobre la escritura en la educación superior emergentes del análisis de reportes escritos por investigadores para un proyecto colombiano interinstitucional desarrollado entre 2009 y 2011 en el cual participé. El análisis de expectativas relacionadas con la escritura, que emergen de reportes escritos por los investigadores participantes en proyectos colegiados recientes, podría ser útil en la comprensión de las diversas agendas de trabajo que se han desarrollado en distintas partes del mundo en el campo de los estudios de la escritura en la educación superior y, desde ellas, prefigurar nuevas agendas de estudios comparados regionales para el progreso del campo como un fenómeno internacional.

\section{METODOLOGÍA}

Este artículo describe expectativas sobre la escritura en la educación superior que son inferidas de documentos escritos producidos en el marco de un proyecto de investigación interuniversitario titulado: "Lectura, escritura y universidad" (LEU). Dado que este trabajo es un estudio de caso, los resultados que se presentan pueden ser considerados generalizaciones teóricas (Yin, 2003), ya que pueden ser agregados a la literatura sobre estudios de la escritura en la educación superior alrededor del mundo (cfr. Bazerman et al., 2012; Thaiss et al., 2012). A continuación se presenta una descripción del proyecto LEU que contextualiza, posteriormente, la descripción de las unidades de análisis, los datos y su tratamiento analítico.

\section{Un proyecto colombiano interinstitucional}

El proyecto de investigación "Lectura, escritura y universidad" (LEU) (Ilevado a cabo entre el año 2009 y el 2011, por diecisiete universidades colombianas) se propuso describir, caracterizar, analizar e interpretar las prácticas de lectura y escritura 
académicas. ${ }^{2}$ Se exploró, cuantitativa y cualitativamente, cómo se lee y escribe principalmente en español, en pregrados presenciales diurnos en distintas áreas de conocimiento; se recogió información de distintas disciplinas y desde las perspectivas de estudiantes, profesores y directivos. El proyecto se ejecutó en las siguientes etapas:

- Etapa 0: discusión colegiada y diseño del proyecto.

- Etapa 1: recolección y análisis de documentos de política institucional relacionados con la lectura y la escritura y programas de asignaturas sobre la enseñanza de las mismas. Análisis cuantitativo de contenidos de los documentos.

- Etapa 2: descripción de prácticas de escritura de 3719 estudiantes de pregrado de distintas disciplinas organizadas de acuerdo con la clasificación de la UNESCO a partir de una encuesta aplicada a estudiantes que habían cursado, como mínimo, el 50\% de los requisitos de sus programas. Análisis a partir de estadística descriptiva.

- Etapa 3: desarrollo y análisis de grupos de discusión, conducidos por separado, con profesores, estudiantes y los investigadores del proyecto LEU.

- Etapa 4: definición, descripción y análisis de "prácticas destacadas en la enseñanza de la escritura" (veinte estudios de caso). Dichos casos fueron inicialmente identificados como parte de la información recogida en la encuesta. Los estudios de caso contaron con la participación de los profesores que aceptaron contribuir de manera voluntaria en el proyecto.

El proyecto LEU estuvo delimitado por tres categorías teóricas: cultura académica, didáctica y prácticas de escritura en la educación superior.

2 El proyecto fue dirigido por el profesor Mauricio Pérez-Abril de la Pontificia Universidad Javeriana de Bogotá D.C. y la profesora Gloria Rincón Bonilla de la Universidad del Valle, los resultados se reportan en la obra ¿Para qué se lee y se escribe en la universidad colombiana? Un aporte a la consolidación de la cultura académica del país. Se encuentra disponible en http://wac.colostate.edu/books/colombian/highered.pdf
La cultura académica es entendida como producción simbólica y cultural enmarcada institucionalmente y asociada a las disciplinas, la escritura (en especial la publicación), las formas de argumentación y las prácticas científicas (Becher, 1993, 2001; Bourdieu, 2008). La didáctica denota el campo disciplinar autónomo desarrollado para investigar y teorizar las interrelaciones entre la enseñanza y el aprendizaje, vistos como procesos escolares sistemáticos, intencionales, histórica e ideológicamente situados (Camps, 2003; 2004). Finalmente, para el proyecto LEU las prácticas de escritura en la educación superior se asocian al aprendizaje y comunicación de los discursos disciplinares; por lo tanto, enseñar la escritura como contenido explícito y diferenciado por disciplinas se considera el camino deseable (Carlino, 2004).

El proyecto LEU también se propuso fortalecer una red nacional de académicos relacionada con la escritura en la educación superior. En consecuencia, el equipo sostuvo dos reuniones anuales presenciales de tres días para negociar y tomar decisiones teóricas y metodológicas. También se utilizó una plataforma digital BSCW (Basic Support for Cooperative Work, por sus siglas en inglés) para publicar datos, documentos de trabajo sobre decisiones teóricas y metodológicas, reportes institucionales de las universidades, resultados nacionales y publicaciones.

\section{Unidad de análisis}

Las unidades de análisis de los datos que son reportados en este artículo se encuentran delimitadas en dos niveles (Merriam, 2009). La primera unidad analítica, el proyecto colombiano e interuniversitario LEU, es una entidad social circunscrita en un periodo de tiempo (2008-2011) y un área geográfica, Colombia (Yin, 2003). Debido a que el área académica de los estudios colombianos de la escritura en la educación superior es emergente (Uribe-Álvarez y Camargo-Martínez, 2011; Ortiz-Casallas, 2011), el proyecto LEU es un caso de cómo dicho campo se ha desarrollado en el país 
(Merriam, 2009), así como un esfuerzo sui generis (Merriam, 2009; Yin, 2003), dada la manera como se creó el equipo de investigación, se negoció y ejecutó el proyecto; por ello, el proyecto LEU es valioso de analizar como un hito en la consolidación de los logros colombianos en el área.

La segunda unidad de análisis se conformó a partir de reportes institucionales escritos por el equipo de investigación de tres universidades distintas de las diecisiete participantes en el proyecto LEU. Los reportes corresponden a la segunda etapa del proyecto (descripción de prácticas de lectura y escritura de 3719 estudiantes encuestados), y a la cuarta etapa (definición, descripción y análisis de "prácticas destacadas en la enseñanza de la escritura"). Por lo tanto, este trabajo se basó en una aproximación comparativa (Merriam, 2009) de los reportes escritos por los investigadores de estas tres universidades distintas y en dos etapas de la investigación. Dichos reportes fueron documentos de trabajo en los que los investigadores organizaban y comentaban los hallazgos de sus propias instituciones.

\section{Datos}

Una muestra intencional y por conveniencia fue creada a partir de reportes institucionales de tres universidades distintas (Merriam, 2009). Los reportes institucionales analizados se seleccionaron porque se encontraban disponibles en la plataforma BSCW, donde se almacenaron los archivos del proyecto LEU. A esto se suma que los colegas investigadores de dichas universidades autorizaron el análisis de sus documentos.

La creación de datos se basó en archivos (Eisenhardt, 1989). Dado que los reportes institucionales analizados no eran documentos públicos, su análisis documenta aspectos del proyecto LEU, que de otro modo no sería posible conocer (Merriam, 2009). En consecuencia, este análisis puede ser visto como un caso revelador (Yin, 2003).

Las tres universidades se seleccionaron debido a sus diferencias institucionales en términos de tiempo de creación, así como las regiones del país en las que se encuentran. Esta decisión se tomó bajo la idea de explorar semejanzas entre las expectativas inferidas de los reportes a pesar de las diferencias institucionales. ${ }^{3}$

La universidad 1 es una universidad pública de 30 años de existencia, cuya misión es educar a los estudiantes en competencias científicas y tecnológicas, a fin de que propongan soluciones a los problemas regionales y nacionales; esta universidad está localizada en la capital del departamento y es una región en el país altamente impactada por el tráfico de drogas y el conflicto interno, especialmente en los años noventa.

La universidad 2 es una universidad pública de 183 años de existencia, cuyo proyecto pedagógico incluye la creación de una cultura de la ciencia, la tecnología, el arte y la enseñanza; la ciudad en la que la universidad está localizada es considerada una de las ciudades más antiguas conservadas en América Latina y el 50\% de su población pertenece a alguna comunidad indígena.

Finalmente, la universidad 3 es una universidad pública de 41 años de existencia, cuya misión es promover la cultura, la investigación científica y la educación profesional para mejorar las situaciones intelectuales, morales y económicas de la región; en el 2010 la región en la que esta universidad está localizada fue denominada por el Ministerio del Interior como área de alto riesgo, razón por la cual las acciones militares fueron fortalecidas. ${ }^{3}$

\section{Análisis de datos}

A partir de la pregunta ¿qué expectativas sobre la escritura en educación superior pueden ser inferidas de los documentos escritos por los investigadores durante el proyecto LEU?, se realizó un análisis textual a partir de la lectura profunda de los seis reportes institucionales de las instituciones

\footnotetext{
3 La información institucional de las páginas web de las universidades así como información de las páginas web de las alcaldías de las ciudades en las que las universidades están localizadas fueron utilizadas para realizar estas descripciones.
} 
seleccionados, con miras a: en primer lugar, describir los contenidos en función de su estructura (p. ej. tipos de subtítulos utilizados, formatos y contenidos desplegados en la subsecciones de los reportes); en segundo lugar, identificar expresiones que manifestaban afirmaciones en las que se valoraba la escritura a través de: 1) adjetivos y adverbios (p. ej. "la formación de investigadores parece ser un objetivo no alcanzable fácilmen$\left.\left.t^{\prime \prime}\right), 2\right)$ preguntas retóricas (p. ej. "¿los textos se reescriben y se vuelven a entregar a los grupos de estudiantes?") y 3) conectores (p. ej. "No obstante, los bajos porcentajes..."). Dicho análisis se realizó explorando los reportes como unidades textuales; esto significa que las inferencias realizadas sobre las valoraciones surgían no solo de conexiones entre ideas en un mismo párrafo, sino entre ideas de diversos párrafos, ya que los contenidos se consideraron como parte de la unidad creada por el tipo de documento "reporte".

\section{RESULTADOS}

El análisis de los reportes ${ }^{4}$ en función de su estructura mostró que a pesar de las diferencias entre los subtítulos (p. ej. preguntas que surgen, discusión, resultados relevantes) y los formatos (p. ej. tablas comparativas o dos secciones: resultados/discusión, diagnóstico/sugerencias) utilizados por los investigadores que los escribieron, los documentos se organizaron bajo una estructura de problemas y soluciones. Por lo tanto, en estas dos categorías se describen a continuación las expectativas inferidas y compartidas entre los reportes sobre la escritura en la educación superior que emergieron de los reportes analizados. Los resultados presentan las expectativas en que coinciden los reportes al compararlos entre universidades y etapas del Proyecto LEU; sin embargo, por motivos de espacio para la presentación de este artículo, dichas expectativas

4 El lector debe recordar que los reportes fueron documentos de trabajo en los que los investigadores organizaban y comentaban los hallazgos de sus propias instituciones. se ilustrarán con fragmentos correspondientes a un solo reporte y a una sola universidad.

\section{Expectativas de la escritura en la educación superior emergentes como soluciones}

1. Los profesores de las disciplinas no enseñan a escribir:

Los reportes institucionales concuerdan en que los profesores no enseñan la escritura como proceso, ya que asumen que sus estudiantes ya saben cómo hacerlo. Se advierte que los profesores de las disciplinas, aunque son usuarios activos y efectivos de la escritura disciplinar de sus campos, no son conscientes de las exigencias de esta. Por otro lado, los reportes afirman que no se enseña la escritura si los profesores no son escritores activos ni efectivos en sus campos académicos. ${ }^{5}$

Esto podría sugerir de parte de los docentes, poco interés en apoyar el proceso de escritura, poco reconocimiento de la necesidad de hacerlo o sentir $q$ no tienen las condiciones necesarias para hacerlo. (falta de tiempo, por ejemplo) $[. . .]^{6}$ ¿qué relación hay entre la publicación de docentes y las demandas de escritura a los estudiantes? FR1UN. ${ }^{\circ} 2 .{ }^{7}$

2. La escritura es usada como un instrumento para repetir los discursos de los profesores:

5 De aquí en adelante fragmentos de los reportes para ilustrar los resultados serán incluidos en formato de cita extensa siguiendo las siguientes convenciones: $\mathrm{FR} \mathrm{N} .^{\circ} \mathrm{UN} .^{\circ}$ (Fragmento del reporte $\mathrm{N} .{ }^{\circ}$ de la universidad $\mathrm{N} .{ }^{\circ}$ ). Los fragmentos se extraen de los reportes originales, la ortografía y la edición original ha sido conservada. Dado que eran documentos de trabajo para uso interno del colectivo de investigación, en algunos fragmentos se observan errores tipográficos, ausencia de concordancia gramatical o estilos de escritura abreviada.

6 El símbolo [...] indica que se ha eliminado información, pero que la idea que sigue hace parte del mismo reporte del que el ejemplo se ha extraído.

7 Se recuerda que los fragmentos se tomaron de reportes institucionales escritos de tres universidades durante la segunda etapa del proyecto LEU (descripción de prácticas de lectura y escritura de 3719 estudiantes encuestados) y la cuarta etapa del proyecto (definición, descripción y análisis de "prácticas destacadas en la enseñanza de la escritura"). 
Los reportes critican que los textos de los estudiantes son vistos como dispositivos que repiten el discurso del profesor. Se afirma que por eso revisar principalmente el contenido de los textos de los estudiantes parece ser una de las prácticas de evaluación más comunes reportadas por los estudiantes encuestados.

Dada la preocupación de los docentes por ver los textos escritos de los estudiantes como portadores de conocimiento, se asume que ellos comparten una visión donde la función de la escritura tiene que ver con decir o mostrar el conocimiento y esto se referencia en el criterio central de la corrección del contenido de los escritos. FR1UN. ${ }^{\circ} 1$.

3. La literatura no es parte de los contenidos de enseñanza para la educación de escritores:

Los reportes manifiestan preocupaciones acerca de la ausencia de la literatura como contenido de enseñanza para educar escritores en la formación universitaria, independiente de los campos de conocimiento. Una de las expectativas inferidas de los reportes es que la enseñanza de la literatura empodera a los estudiantes como escritores, para reaccionar y criticar los contextos nacionales y regionales.

En correspondencia con lo anterior los temas sobre los que menos escriben ambos actores — por actores se está haciendo referencia a estudiantes y profesores—, ${ }^{8}$ son los temas literarios, sociales y políticos. Esta información resulta significativa cuando se considera que la universidad debe llenar de sentido los fenómenos sociales y políticos del contexto local, regional, nacional e internacional, enriqueciéndolos desde la reflexión y la discusión. FR1UN. ${ }^{\circ}$.

4. Las universidades no enseñan cómo escribir artículos científicos:

8 El inciso indica que la investigadora añade aclaraciones para dar más contexto a los fragmentos que ilustran el análisis.
Los reportes concuerdan, de acuerdo con los resultados de la encuesta aplicada a los estudiantes, que es escasa la práctica de escritura de artículos científicos en las universidades participantes del proyecto LEU. Los reportes coinciden en que la baja frecuencia de escritura de artículos científicos por parte de los estudiantes puede perjudicar el progreso científico del país.

Refieren —los estudiantes- escribir muy poco artículos científicos, lo que podría ocasionar que los futuros profesionales estén incapacitados para producir el tipo de documento exigido para la publicación en revistas de reconocimiento para una comunidad académica determinada. FR1UN. ${ }^{\circ} 2$.

5. Las experiencias de escritura asociadas con prácticas de investigación son emergentes en las universidades:

Los reportes coinciden en su preocupación por la escasa enseñanza de la escritura científica. Uno de los reportes de la universidad N. ${ }^{0} 1$ hace énfasis en las consecuencias de este hecho para la formación de investigadores en la educación colombiana. La preocupación manifestada en los reportes analizados de la etapa 2 se justificaba porque los resultados de la encuesta recogían información de estudiantes que habían completado como mínimo el $50 \%$ de sus programas de pregrado; por lo tanto, los reportes indicaban que la poca presencia de escritura de artículos científicos en las experiencias de los estudiantes encuestados era preocupante, teniendo en cuenta que habían cursado, como mínimo, la mitad de sus programas.

La vinculación de los escenarios de enseñanza y aprendizaje de los conocimientos disciplinarios y profesionales y los eventos académicos en los cuales se puedan presentar ponencias sobre tópicos específicos parece ser una relación débil, eventual, para estudiantes de carrera que ya superan la mitad de ese recorrido. FR1UN. ${ }^{\circ} 1$. 
6. La escritura es utilizada principalmente como una "actividad escolar":

Los reportes de las universidades coinciden en la preocupación de que los estudiantes escriben principalmente para cumplir con las demandas de los profesores; en otras palabras, los estudiantes escriben solo para cumplir con sus tareas. El reporte institucional sobre la etapa 2 del proyecto de la universidad 3 afirma que la alta frecuencia de tipos de documentos, como las notas de clase, ${ }^{9}$ es un indicador negativo y una posible evidencia de la presencia de prácticas pedagógicas tradicionales basadas en exposiciones por parte del profesor.

A manera de explicación tener un lugar tan destacado la toma de apuntes puede configurarse como indicio de unas prácticas pedagógicas donde prima la educación tradicional, en la que el docente ocupa un lugar central y protagónico pues es él el depositario de la información. FR1UN. ${ }^{\circ} 3$.

El mismo reporte establece la diferencia entre dos nociones: a) "prácticas reales de escritura" (p. ej. escritura de artículos para revistas), y b) "prácticas escolares de escritura" (p. ej. escritura de exámenes, resúmenes y talleres).

7. Publicar y participar en eventos académicos son prácticas escasas para promover experiencias de escritura en los estudiantes:

Los reportes coinciden en su preocupación, debido a que diseñar proyectos, asistir a eventos, publicar ponencias y artículos en revistas son prácticas escasas según los resultados de la encuesta aplicada a los estudiantes. Los reportes concuerdan en que este tipo de experiencias, más allá de las experiencias de escritura en los salones de clase, permite a los estudiantes enfrentar distintos desafíos de escritura.

9 Ver Anexo 1.
La formación de investigadores parece ser un objetivo no alcanzable fácilmente en este nivel, en razón, por un lado, de la poca importancia atribuida a escenarios académicos como los semilleros de investigación —semilleros de investigación es una iniciativa de formación de jóvenes investigadores del sistema universitario colombiano- y la participación en eventos académicos; y, por otro, a la lectura y producción de artículos científicos. FR1UN. ${ }^{\circ} 1$.

8. Las prácticas de escritura para la investigación difícilmente se presentan en espacios distintos a las aulas de clase:

Los reportes coinciden en que la escritura no debería ser usada solo para evaluar a los estudiantes en los salones de clase. Se sugiere la importancia de utilizar la escritura para participar en ámbitos distintos a los que se inscriben en práctica de evaluación.

Llama la atención lo poco que se escribe para redactar ponencias o para publicar, lo que podría ocasionar escasa competencia de los estudiantes para contribuir al avance de su disciplina mediante la socialización de productos propios. FR1U N. ${ }^{\circ} 2$.

\section{Síntesis}

Los anteriores problemas inferidos de los reportes de dos etapas del Proyecto LEU y de tres universidades distintas sugiere que parte de las expectativas sobre la escritura en la educación superior es la formación de los estudiantes como escritores para la investigación y la producción científica; a pesar de que también hay una expectativa sobre la presencia de la literatura como parte de la educación de escritores para la participación crítica en las situaciones nacionales y locales desde su perspectiva como ciudadanos.

El análisis también sugiere que hay una expectativa por explorar las posibles relaciones entre las prácticas de escritura de los profesores, como escritores académicos que publican en sus campos 
y las prácticas de enseñanza con los estudiantes. Una hipótesis que emerge del análisis es que habría impactos positivos o negativos en las prácticas de enseñanza de acuerdo con: i) la conciencia que tienen o no los profesores sobre sus prácticas como escritores académicos, y ii) su participación activa o no como escritores de sus campos. También emerge como hipótesis que la escritura en la universidad no es solo un medio de evaluación y se aboga por la presencia de experiencias de socialización académica fuera de los salones de clase (p. ej. eventos académicos).

A continuación se describen las expectativas inferidas y compartidas entre los reportes que emergieron como soluciones.

\section{Expectativas de la escritura en la educación superior emergentes como soluciones}

1. Los profesores de las disciplinas necesitan formación para la enseñanza de la escritura:

Los reportes institucionales concuerdan en la necesidad de que los profesores de las disciplinas reconozcan campos teóricos que denominados lingüística textual, pragmalingüística y alfabetización académica, así como el uso de la escritura con fines didácticos. Esta formación teórica se considera una solución para enfrentar los problemas que son destacados por los reportes.

En este sentido, las posibilidades didácticas de la función epistémica de la producción escrita y la relevancia de la alfabetización académica (Carlino, 2004) parecen caminos de solución que debieran ser puestos en conocimiento de los docentes de las diferentes áreas disciplinarias que participan en la vida académica de cada universidad, en el ámbito nacional como en el caso particular de la Universidad N. ${ }^{\circ} 1$. FR1UN. ${ }^{\circ} 1$.

2. Las prácticas de enseñanza basadas en una perspectiva didáctica son necesarias para educar escritores en la universidad como profesionales y ciudadanos:
Los reportes institucionales manifiestan la defensa de las siguientes agendas en las universidades: a) la educación de profesionales y ciudadanos entendidos como agentes sociales; $y$, b) el lugar de la Didáctica como una estrategia en la consecución de dicha meta educativa.

Finalmente, en la perspectiva de incorporar los hallazgos de la investigación a la práctica social que permitan concretar el carácter humanista y transformador de la didáctica, se desea expresar el convencimiento de que las prácticas de lectura y escritura de los estudiantes no son su exclusiva responsabilidad, sino que son el resultado de las múltiples determinaciones en las que intervienen las instituciones educativas, sus instancias colegiadas, y los docentes. Sobre ese paisaje de compromiso compartido es posible imaginar el desarrollo de cada estudiante, en primer lugar, para comprender su formación como actor constituido desde su formación disciplinaria y profesional; y, en segundo lugar, para ejercer la crítica y la transformación personal y social desde esa misma formación. FR1UN. ${ }^{\circ} 1$.

3. La escritura debe ser enseñada como un aprendizaje para toda la vida:

Los reportes institucionales concuerdan en que la enseñanza de la escritura debe basarse en actividades de reflexión y meta-evaluación porque incrementan la conciencia y duración de los aprendizajes. Uno de los reportes sobre prácticas destacadas de la enseñanza de la lectura y la escritura valora positivamente cómo un profesor de leyes ofrece apoyo a los estudiantes para leer y escribir mientras se prepara una ponencia que los estudiantes presentarán en un evento.

El docente contextúa los procesos formativos presentándoles a los estudiantes los hechos relevantes. Luego los sintetiza, se hace la lectura; se hace consciencia sobre la complejidad de los temas. Se hace un análisis conjunto del proceso incluyendo 
prácticas de meta-evaluación. Se corrige colectivamente y se reelabora la ponencia. FR2UN. ${ }^{\circ} 1$.

4. Las experiencias positivas de aprendizaje incorporan actividades de escritura cuyas audiencias son distintas al profesor:

Los reportes concuerdan que escribir para audiencias distintas al profesor es una práctica clave de la enseñanza de la escritura y justifican que, bajo estas circunstancias, los estudiantes podrán tomar decisiones y hacer cambios en sus textos a partir de las demandas y expectativas de lectores reales con roles externos no ligados a las experiencias educativas. Uno de los reportes sobre las prácticas destacadas de enseñanza de los profesores subraya la importancia del rol de un profesor en historia que lee los trabajos de los estudiantes para evaluarlos desde el punto de vista del comité científico que elegirá ponencias de estudiantes para participar en un evento académico.

Esta experiencia académica se identifica como experiencia destacada, no solo por la trayectoria del docente, es decir su perfil académico e investigativo sino desde su visión propia en la formación de historiador. Esta perspectiva, intrínseca en el docente le lleva a trascender el aula de clases, trasciende los límites del aula y de la evaluación docente. El evaluador no solamente es el docente sino un Comité especializado que selecciona las ponencias en un evento internacional. FR2UN. ${ }^{\circ} 2$.

5. Los textos complejos (comentarios de textos, ensayos y artículos de la profesión) deben ser enseñados por los profesores:

Los reportes institucionales concuerdan en que ciertos tipos de textos demandan procesos cognitivos más complejos que otros. Por ejemplo, uno de los reportes clasifica los textos de acuerdo con el grado en que los escritores deben incluir o no sus puntos de vista. En consecuencia, las notas de clase, los resúmenes y los reportes se denominan en este reporte textos expositivos, porque los escritores no están obligados a incluir sus perspectivas respecto de los temas que escriben; en consecuencia, este reporte denomina a dicho tipo de textos como documentos que incluyen contenidos referenciales. Por otro lado, los ensayos son vistos como documentos que ofrecen oportunidades de aprendizaje complejo porque se espera que los escritores incluyan sus propias perspectivas sobre el tema que escriben. Como resultado, los ensayos son tipos de documentos que se destacan en los reportes como textos deseables para la enseñanza de la escritura en las disciplinas.

Uno de los reportes de la universidad 3, aunque también manifiesta que hay tipos de textos académicos que son más fáciles que otros, enfatiza en que el grado de complejidad depende de los rasgos lingüísticos de los textos así como la inclusión o no del punto de vista del escritor. Este mismo reporte sugiere que textos académicos como comentarios, ensayos, reportes y artículos serán siempre complejos al ser escritos por practicantes de un campo académico.

Lo anterior — se hace referencia a la necesidad de enseñar a los estudiantes las prácticas de escritura propias de la educación superior y el mundo académico- exige intentar establecer qué se entiende por texto académico. Al respecto Ana Teberosky y Moserrat Castellò (2007) sugieren que lo importante, no es la elección de una modalidad de texto en particular como la reseña, el ensayo, el artículo, etc., sino el cumplimiento de unas características e intencionalidades que éste comporta. En tal caso un texto académico es en consecuencia un desarrollo consciente de adquisición de conocimiento, de exposición y debate del pensamiento, pero sobre todo la formación de un criterio propio. Esto implica un proceso complejo que involucra el estudio de la situación retórica, en el que su lector será otro miembro de la comunidad académica, donde el texto debe regirse por unos cánones que 
determinan que es lo apropiado o lo no apropiado en el contexto, y en el que su finalidad es argumentar, convencer o persuadir. FR2UN. ${ }^{\circ} 3$.

6. La reescritura debe ser parte de la enseñanza:

La reescritura es asociada en los reportes con prácticas positivas para la enseñanza de la escritura. Los reportes coinciden en que el uso de la reescritura durante la enseñanza es un indicio de que los profesores entienden la escritura como un proceso. Uno de los reportes de la universidad 1 sostiene que es más probable que dicha práctica sea llevada a cabo por profesores de las disciplinas que tienen una conciencia discursiva.

Del mismo modo, uno de los reportes de la universidad 2 afirma que si la enseñanza incluye la escritura como proceso, el profesor llevará a cabo actividades de escritura en las que no se espera un texto final definitivo. En ese reporte se dice que aunque los profesores tengan una conciencia sobre la necesidad de la reescritura podrían no incluir esta práctica en su enseñanza porque consideran que requieren de mucho tiempo y es demandante.

Esto podría sugerir —el reporte está haciendo referencia a resultados de la encuesta que parecen indicar que los textos de los estudiantes no son leídos o discutidos en clase- de parte de los docentes, poco interés en apoyar el proceso de escritura, poco reconocimiento de la necesidad de hacerlo o sentir q no tienen las condiciones necesarias para hacerlo (falta de tiempo, por ejemplo). FR1UN. ${ }^{\circ} 2$.

\section{La escritura debe ser enseñada como proceso:}

Los reportes concuerdan en la importancia de las siguientes prácticas de enseñanza: a) los documentos de los estudiantes deben experimentar cambios durante una experiencia de aprendizaje; b) los documentos de los estudiantes deben ser leídos en clase para analizarlos de acuerdo con criterios explícitos de evaluación; c) los estudiantes deben ser pares revisores durante las prácticas de evaluación; y, d) los profesores no deben ser los únicos revisores y asesores de los textos de los estudiantes.

La encuesta no permite llegar a afirmaciones concluyentes acerca de las condiciones propias del circuito comunicativo que sufren los textos escritos por los estudiantes. Por ejemplo, no se puede decir qué pasa con los textos de los estudiantes leídos en clase, corregidos en su contenido: ise re-escriben y vuelven a presentarse a los grupos? ¿Estos mismos textos son los que se entregan para que sean leídos y evaluados, fuera de la clase, por los docentes? [...] ¿No podrían los estudiantes, en una labor de co-evaluación, legitimar la validez disciplinaria de un texto producido por otro compañero? FR1U N. ${ }^{\circ} 1$

8. Los profesores de las disciplinas deben abordar objetivos pedagógicos explícitos de los contenidos disciplinares que están relacionados con la escritura porque la escritura no es una meta en sí misma:

Los reportes expresan que la escritura está incrustada en las disciplinas y profesiones, lo que significa que no es una meta en sí misma. Los reportes resaltan la importancia de que los profesores y los estudiantes identifiquen rutinas instruccionales en las que la escritura sea necesaria para aprender y practicar los contenidos de las asignaturas; además se recalca que dichas actividades deben anticipar las situaciones prácticas que los estudiantes enfrentarán en su futuro profesional. Una de las prácticas valoradas positivamente en uno de los reportes de la universidad 3 destaca las opiniones de los estudiantes sobre las oportunidades de aprendizaje que les dio una experiencia de enseñanza en ingeniería de sistemas.

El profesor es estricto - este es un fragmento que parafrasea comentarios de los estudiantes sobre este profesor- pero brinda toda la ayuda 
necesaria. Él es excelente y disciplinado (cree que somos muy chillones), pasamos la asignatura muy arrastrados pero luego valoramos lo que quería de nosotros. Él desde el comienzo del semestre platea qué es lo que se va a realizar y efectivamente se realiza. Desde el comienzo se sabe cómo vamos a ser evaluados y los criterios. Estas prácticas deberían ser asumidas por otras asignaturas porque el ejercicio de escribir exige mucho análisis y síntesis, obliga a pensar antes de escribir. FR2UN. ${ }^{\circ}$.

\section{Síntesis}

Las expectativas que emergen como soluciones de los reportes de dos etapas del Proyecto LEU y de tres universidades distintas abogan por la formación de profesores universitarios, de modo que sean preparados para liderar experiencias de escritura en la educación de profesionales y ciudadanos caracterizadas por i) la reescritura; ii) la producción de textos académicos complejos dirigidos a audiencias distintas, no solo al profesor, o con roles externos a las situaciones educativas inmediatas en las que se escribe; iii) actividades explícitas de metaevaluación; y, iv) la discusión de los textos de los estudiantes durante las clases.

\section{DISCUSIÓN}

El mapa de problemas y soluciones identificado es útil para describir qué es criticado y valorado en relación con la escritura en la educación superior en un proyecto colombiano interinstitucional; este análisis se convierte en una ventana para explorar las expectativas de académicos relacionados con este campo en Colombia y sus agendas de trabajo. Las tendencias identificadas permiten proponer las siguientes agendas pedagógicas y de investigación asociadas con el análisis realizado como parte de las iniciativas que podrían estarse liderando en Colombia.
Como agendas pedagógicas se encontrarían:

1. Diseño y ejecución de programas de formación de profesores de todas las disciplinas sobre la enseñanza de la escritura desde:

- i) perspectivas didácticas para producir textos que tienen nuevas funciones además de las evaluativas, y

- ii) perspectivas teorías denominadas en los reportes como lingüística textual, la pragmalingüística y la alfabetización académica.

2) Fortalecimiento de iniciativas acerca de la escritura académica, profesional y para la ciudadanía, llevadas a cabo fuera de las aulas de clase; y

3) Asesorías sobre escritura de artículos científicos y comunicaciones orales para grupos de investigación que forman jóvenes investigadores.

En relación con las agendas de investigación podrían encontrarse:

1) Investigaciones para conceptualizar diferencias entre lo que se ha Ilamado "textos académicos simples y complejos" y "prácticas reales de escritura y prácticas escolares de escritura", así como su interrelación con procesos de aprendizaje de las disciplinas, en general, y de la escritura, en particular;

2) Investigaciones para evidenciar las diferencias y articulaciones entre programas de formación de escritores para la ciudadanía, la producción académica y profesional, por ejemplo las hipótesis relacionadas con el impacto de la literatura en la educación de ciudadanos y el desarrollo del pensamiento crítico;

3) investigaciones que exploren las hipótesis de interrelación entre las prácticas de publicación de los profesores, su conciencia disciplinar sobre 
la escritura en su campo académico y las prácticas de enseñanza que lideran;

4) Investigaciones para explorar los impactos de las agendas pedagógicas descritas anteriormente y que se hayan emprendido en las universidades colombianas.

Este análisis muestra dos finalidades en la formación de escritores en la educación superior para el caso colombiano: para la profesionalización y el mundo académico, y para la ciudadanía (problema N. ${ }^{\circ} 3$ y solución N. ${ }^{\circ}$ 2). Su coexistencia podría justificarse en los contextos sociales y políticos en los que se inscriben las universidades de los reportes analizados. Por otra parte, emerge una fuerte defensa para la formación de escritores con experticia para la publicación científica e investigativa (problemas N. ${ }^{\circ} 4,5,7,8$ ) que se justifica en la necesidad de progreso científico en el contexto colombiano.

Al poner en contraste estas agendas de intervención y de investigación con la descripción de otras agendas a nivel internacional del campo de los estudios de la escritura, se podría decir que los movimientos sobre la escritura a través del currículo (WAC) y la escritura en las disciplinas (WID) originalmente desarrollados en EEUU (cfr. Russell, Lea, Parker y Donahue, 2009) serían paradigmas desde los cuales se enmarca la mayoría de las expectativas presentadas en este reporte (problemas N. ${ }^{\circ} 1,2,6$ y soluciones N. ${ }^{\circ} 1,6,7$ y 8). Esta tendencia se explica también en el marco teórico que adoptó el proyecto LEU y muestra que los reportes se encuentran alineados con dicha perspectiva conceptual.

También se observa una influencia de paradigmas franceses, especialmente en relación con el análisis textual para hacer inferencias sobre las complejidades de los textos académicos (solución N.․ 3), aunque ya se ha afirmado que esta aproximación es importante pero no es suficiente en la comprensión de las prácticas de enseñanza de la escritura en la educación superior (cfr. Donahue, 2009).
En contraste con agendas internacionales, no surgen de este análisis la reflexión e investigación sobre prácticas de escritura que enfrentan poblaciones estudiantiles que se han denominado como "no tradicionales" (cfr. Lillis, 2003) y prácticas académicas de escritura y de enseñanza emergentes, como las relacionadas con las tecnologías digitales (cfr. Thaiss, Zawacki y Myers, 2006).

Al comparar el análisis con la sistematización de experiencias latinoamericanas y colombianas (cfr. Uribe-Álvarez y Camargo-Martínez, 2011; Ortiz-Casallas, 2011), se observa que temas como la investigación sobre los cursos de escritura de primer año, una iniciativa curricular fuertemente presente en la región (cfr. Proyecto ILEES) y la exploración de las condiciones que favorecen la sostenibilidad de las iniciativas como parte de políticas institucionales (cfr. Quintero y Duque, 2011; Rincón y Gil, 2010; Rincón y Pérez, 2011; Carlino, 2012) serían temas relevantes de trabajo en la región.

Esta configuración de las agendas locales en relación con otras realizadas alrededor del mundo es un importante contraste para insistir en cómo los estudios de la escritura en la educación superior están influenciados por los sistemas universitarios en los que se han desarrollado, así como para prefigurar nuevas agendas de estudios comparados regionales para el progreso del campo como un fenómeno internacional.

\section{CONCLUSIÓN}

Este proyecto exploratorio tuvo por objetivo analizar las expectativas inferidas de reportes de investigación escritos y divulgados al interior de un grupo colombiano de investigación interinstitucional entre el año 2009 y el año 2011. Dicho análisis aporta a la sistematización de los esfuerzos en el caso colombiano en la consolidación de una comunidad emergente sobre la práctica y la investigación sobre los estudios de la escritura en la educación superior. La identificación de expectativas compartidas a través de la comparación de seis reportes institucionales de tres universidades distintas y 
derivados de dos etapas de la investigación interinstitucional seleccionada como caso ofrece un mapa de problemas y soluciones que puede verse como una descripción de posibles agendas de práctica y de investigación que se están adelantando en el país. La sistematización y divulgación de análisis de expectativas emergentes de investigaciones sobre la escritura en la educación superior es una práctica importante en la consolidación y proyección de la comunidad de académicos que están participando de esta discusión en los últimos años para el caso colombiano y como datos por agregar a las sistematizaciones internacionales sobre los estudios de la escritura.

\section{RECONOCIMIENTOS}

Dado que este artículo surge del análisis de los reportes escritos por investigadores que participaron en un proyecto colombiano interinstitucional desarrollado entre el año 2009 y el año 2011 sobre lectura y escritura académica (el proyecto LEU), agradezco a los colegas que permitieron el análisis de los documentos tratados como datos y a los directores del Proyecto LEU que aceptaron el uso de dichos archivos. También agradezco a los pares evaluadores cuyas preguntas me ayudaron a repensar la mejor manera de reportar este análisis.

\section{REFERENCIAS}

Bazerman, C., Dean, Ch., Early, J., Lunsford, K., Null, S., Rogers, P., \& Stansell, A. (Eds.). (2012). International Advances in Writing Research: Cultures, Places, Measures. Perspectives on Writing Series. Fort CoIlins, Colorado: The WAC Clearinghouse and Parlor Press. Recuperado de http://wac.colostate.edu/ books/wrab2011/

Becher, T. (1993). Las disciplinas y la identidad de los académicos. Pensamiento universitario, 1(1), 56-77. Recuperado de http://inter27.unsl.edu.ar/ rapes/?action=detalle $\&$ from $=$ todos $\& i d=441$

Becher, T. (2001). Tribus y territorios académicos. Barcelona: Gedisa.
Bourdieu, P. (2008). Homo academicus. Buenos Aires: Siglo XXI.

Camps, A. (2004). Objeto, modalidades y ámbitos de la investigación en didáctica de la lengua. Lenguaje, 32, 7-27.

Camps, A. (Comp.). (2003). Secuencias didácticas para aprender a escribir. Barcelona: Graó.

Carlino, P. (2004). Escribir y leer en la universidad: responsabilidad compartida entre alumnos, docentes e instituciones. En P. Carlino (Coord.). Leer y escribir en la Universidad. Serie Textos en Contexto, 6 (5-21). Buenos Aires: Asociación Internacional de Lectura, Lectura y Vida.

Carlino, P. (2012). Section essay: Who takes care of writing in Latin American and Spanish universities? En Chris Thaiss, Gerd Bräuer, Paula Carlino, Lisa Ganobcsik-Williams, \& Aparna Sinha (Eds.). Writing Programs Worldwide: Profiles of Academic Writing in Many Places. Perspectives on Writing, 485. Fort Collins, Colorado: The WAC Clearinghouse and Parlor Press. Recuperado de http://wac.colostate.edu/books/wrab2011/

Donahue, T. (2009). Genre and disciplinary work in French didactics research. En C. Bazerman, A. Bonini, \& D. Figueiredo. Genre in a Changing World (424-441). Perspectives on Writing Series. Fort Collins, Colorado: The WAC Clearinghouse and Parlor Press. Recuperado de http://wac.colostate. edu/books/genre/

Eisenhardt, K. (1989). Building theories from case study research. Academic of Management Review, 14(4), 532-550.

ILEES (s.f.). El proyecto ILEES: hacia un mapa exhaustivo de la escritura en la educación terciaria en la región. Recuperado de http://www.ilees.org

Lillis, T. (2003). Student writing as 'Academic Literacies': Drawing on Bakhtin to move from critique to design. Journal Language and Education, 17(3), 192-201. Recuperado de http://www.writing. ucsb.edu/wrconf08/Pdf_Articles/Lillis_Article2. pdf

Merriam, S. B. (2009). Qualitative Research: A Guide to Design and Implementation. San Francisco: Jossey-Bass. 
Ortiz-Casallas, E. M. (2011). La escritura académica universitaria: estado del arte. Íkala, Revista de lenguaje y cultura, 16, 17-41. Recuperado de http://redalyc.uaemex.mx/src/inicio/ArtPdfRed. jsp?iCve $=255019720002$

Pérez-Abril, M. y Rincón-Bonilla, G. (Coords.) (2013). ¿Para qué se lee y se escribe en la universidad colombiana? Un aporte a la consolidación de la cultura académica del país. Bogotá: Editorial Pontificia Universidad Javeriana. Recuperado de http://wac.colostate.edu/books/ colombian/

Quintero, G. y Duque, C. (2011). La lectura y la formación profesional. Hechos y Proyecciones del Lenguaje, 19, 276-298.

Rincón, G. y Pérez, M. (2011). Para qué se lee y se escribe en la universidad colombiana: Un aporte a la cultura académica del país (PRE00439015708). Bogotá: Colciencias.

Rincón, G. y Gil, J. (2010). Las prácticas de lectura y de escritura académicas en la Universidad del Valle: tendencias. Lenguaje, 38, 319-387.

Russell, D. \& Yañez, A. (2003). 'Big Picture People Rarely Become Historians': Genre Systems and the Contradictions of General Education. En C. Bazerman \& D. Russell (Eds.). Writing Selves/Writing Societies: Research from Activity Perspectives (331-362). Perspectives on Writing Series. Fort Collins, Colorado: The WAC Clearinghouse. University of California San
Diego, La Jolla, California: Mind, Culture, and Activity: An International Journal. Recuperado de http:// wac.colostate.edu/books/selves_societies/russell/

Russell, D. R. (2013). Contradictions regarding teaching and writing (or writing to learn) in the disciplines: What we have learned in the USA. Revista de Docencia Universitaria, 11(1), 161-181.

Russell, D., Lea, M., Parker, J., Street, B. \& Donahue, T. (2009). Exploring notions of genre in "Academic Literacies" and "Writing across the Curriculum": Approaches across countries and contexts. En C. Bazerman, A. Bonini, \& D. Figueiredo. Genre in a Changing World (395-423). Perspectives on Writing Series. Fort Collins, Colorado: The WAC Clearinghouse and Parlor Press. Recuperado de http:// wac.colostate.edu/books/genre/

Thaiss, C., Zawacki, T. Myers. (2006). Engaged Writers and Dynamic Disciplines: Research on the Academic Writing Life. Portsmouth, NH: Boynton/Cook, Heinemann.

Uribe-Álvarez, G. y Camargo-Martínez, Z. (2011). Prácticas de lectura y escritura académicas en la universidad colombiana. Magis, Revista Internacional de Investigación en Educación, 3(6), 317-341. Recuperado de http://www.javeriana.edu.co/magis/numero-seis/pdfs/magis-3-6-Graciela-Uribe.pdf

Yin, R.K. (2003). Case Study Research: Design and Methods. Thousand Oaks, California: Sage. 
Anexo 1. Resultado asociado a la pregunta $N .^{\circ} 4$ de la encuesta

¿Con qué propósitos escribe en las actividades académicas seleccionadas en la pregunta 2? (Puede marcar varias opciones)

Diseñar un proyecto

Elaborar una relatoría

Redactar ponencias

Presentar informes

Responder a una evaluación escrita

Elaborar escritos para una exposición (guías, resúmenes, acetatos, diapositivas, etc.)

Elaborar notas personales

Escribir artículos para ser publicados

Otros ¿Cuál? 